\title{
Nontoxic hydrophilic polymeric nanocomposites containing silver nanoparticles with strong antimicrobial activity
}

This article was published in the following Dove Press journal:

International Journal of Nanomedicine

31 March 2016

Number of times this article has been viewed

\author{
Alexander S Pozdnyakov' \\ Artem I Emel'yanov' \\ Nadezhda P Kuznetsova' \\ Tamara G Ermakova' \\ Tat'yana $V$ Fadeeva ${ }^{2}$ \\ Larisa M Sosedova ${ }^{3}$ \\ Galina F Prozorova' \\ 'AE Favorsky Irkutsk Institute of \\ Chemistry, Siberian Branch, Russian \\ Academy of Sciences, ${ }^{2}$ Federal State \\ Budgetary Scientific Institution \\ Irkutsk Scientific Center of Surgery \\ and Traumatology, Laboratory of \\ Functional Genomics and Interspecific \\ Interactions of Microorganisms, \\ Irkutsk, ${ }^{3}$ East-Siberian Institute \\ of Medical \& Ecological Science, \\ Department of Biomodeling \& \\ Translational Investigation, Angarsk, \\ Russia
}

\begin{abstract}
New nontoxic hydrophilic nanocomposites containing metallic silver nanoparticles (AgNPs) in a polymer matrix were synthesized by the chemical reduction of silver ions in an aqueous medium. A new nontoxic water soluble copolymer of 1-vinyl-1,2,4-triazole and $\mathrm{N}$-vinylpyrrolidone synthesized by free radical-initiated polymerization was used as a stabilizing agent. Transmission electron microscopy, scanning electron microscopy, ultraviolet-visible spectroscopy, Fourier transform infrared spectroscopy, X-ray diffraction, atomic absorption, and thermogravimetric analysis were used to characterize polymeric AgNPs nanocomposites. The results showed that the diameter of the synthesized AgNPs ranged from 2 to $6 \mathrm{~nm}$. The toxicity of the initial copolymer of 1-vinyl-1,2,4-triazole and $\mathrm{N}$-vinylpyrrolidone and its nanocomposite with AgNPs was found to be more than $5,000 \mathrm{mg} / \mathrm{kg}$. The synthesized AgNP polymeric nanocomposite showed significant antimicrobial activity against different strains of Gram-negative and -positive bacteria. The minimum inhibitory concentrations suppressing the growth of the microorganisms ranged from 0.5 to $8 \mu \mathrm{g} / \mathrm{mL}$ and the minimum bactericidal concentrations ranged from 0.5 to $16 \mu \mathrm{g} / \mathrm{mL}$. The fabricated AgNP nanocomposites are promising materials for the design of novel nontoxic hydrophilic antiseptics and antimicrobial components for medical purposes.
\end{abstract}

Keywords: nontoxic nanocomposites, antimicrobial activity, silver nanoparticles, 1-vinyl-1,2,4triazole, N-vinylpyrrolidone

\section{Introduction}

The design of modern medicines for the treatment of diverse diseases, including socially important ones (tuberculosis, HIV, AIDS, cancer, cardiovascular disorders, etc), strongly depends upon the development of novel functional polymer nanocomposite materials possessing a variety of valuable properties. Such materials are extensively applied in medicinal chemistry and pharmacology (biologically active compounds and systems of targeted delivery of contrasting agents and drugs). ${ }^{1-3}$ Among the essential properties of these materials are hydrophilicity, pharmacological activity, nontoxicity, and resistance toward aggressive media. The presence of functional groups allowing the binding with different drugs and contrasting agents is also very important.

The above properties are inherent in 1-vinyl-1,2,4-triazole-based polymers, which represent promising, though poorly studied, compounds of medicinal application. Poly-1-vinyl-1,2,4-triazole is a unique biocompatible hydrophilic nontoxic (lethal dose, $50 \%\left[\mathrm{LD}_{50}\right]>5,000 \mathrm{mg} / \mathrm{kg}$ ) polymer with chemical stability in concentrated acids and alkalis, and thermal stability up to $340^{\circ} \mathrm{C}$ (on air), and has complexation and quaternization properties. Its polymers are promising in terms of the design of
Correspondence: Alexander S Pozdnyakov

AE Favorsky Irkutsk Institute of Chemistry, I Favorsky Street, 664033 Irkutsk, Russia

$\mathrm{Tel}+73952425531$

$\mathrm{Fax}+73952419346$

Email as_pozdnyakov@mail.ru (c) (1) (5) 2016 Pozdnyakov et al. This work is published and licensed by Dove Medical Press Limited. The full terms of this license are available at https://www.dovepress.com/terms.php cc) and incorporate the Creative Commons Attribution - Non Commercial (unported, v3.0) License (http://creativecommons.org/licenses/by-nc/3.0/). By accessing the work you hereby accept the Terms. Non-commercial uses of the work are permitted without any further permission from Dove Medical Press Limited, provided the work is properly attributed. For permission for commercial use of this work, please see paragraphs 4.2 and 5 of our Terms (https://www.dovepress.com/terms.php). 
modern biologically active products for medical applications, such as materials for soft contact lenses, biosynthetic activation of connective tissue cells, sorbents for chromatographic purification of viruses, latexes, and other colloidal systems, and agents able to prolong the action of some drugs. Recently, it has been found that polymers of 1-vinyl-1,2,4triazole represent a highly effective polymeric stabilizing matrix for metallic nanoparticles, and the nanocomposites obtained show properties of polymer and metal counterparts (hydrophilicity, complex formation ability, chemical thermal stability, biological activity, etc). ${ }^{4-7}$ Also, it has been demonstrated $^{8}$ that polymer nanocomposites containing silver nanoparticles (AgNPs) in the poly-1-vinyl-1,2,4-triazole matrix exert antimicrobial activity against Gram-positive and -negative museum and hospital strains, nonfermenting bacteria, and fungi.

Nanocomposites based on polymers of N-vinylpyrrolidone with AgNPs have found wide application in medicine. For example, antiseptics poviargolum, collargolum, and so on are used in traumatic surgery (purulent inflammatory wound infections), burn injury medicine (various infectious burns), otolaryngology (rhinitis, sinusitis, pharyngitis, otitis), ophthalmology (purulent conjunctivitis, keratitis), and so on. ${ }^{9,10}$ The antimicrobial activity of polymer nanocomposites increases with decreasing size of AgNPs, so approaches to the preparation of small AgNPs (on average up to $10 \mathrm{~nm}$ ) are attracting increasing attention of researchers. ${ }^{11}$

It is known that metallic nanoparticles have a high surface area-to-volume ratio resulting in their aggregation with time that limits their potential applications. ${ }^{12-15}$ Therefore, it is of special importance that the stabilizing matrix could efficiently stabilize metal nanoparticles preserving their properties for a long period of time. This is achieved by the usage of a polymer matrix containing different functional groups, which are absorbed on the surface of AgNPs thus preventing their aggregation. In addition, functional groups significantly expand application areas of polymer nanocomposites, ensuring their binding with diverse drugs and contrasting agents.

In this paper, we report on the synthesis and characterization of nanocomposites containing AgNPs in a new nontoxic matrix of copolymer of 1-vinyl-1,2,4-triazole and N-vinylpyrrolidone (poly (VT-co-VP)) $\left(\mathrm{LD}_{50}>5,000 \mathrm{mg} / \mathrm{kg}\right) . \mathrm{We}$ discuss the results of this research with regard to the main physicochemical properties and antimicrobial activity of these nanocomposites. The toxicological properties of the starting poly (VT-co-VP) and nanocomposite containing AgNPs are also studied. New polymer nanocomposites synthesized from functional poly (VT-co-VP) can extend the assortment of nontoxic hydrophilic polymeric materials, which opens up new prospects for their use in medicine.

\section{Materials and methods Materials}

The starting 1-vinyl-1,2,4-triazole (boiling temperature $\left.43^{\circ} \mathrm{C} / 3 \mathrm{mmHg}, \mathrm{n}_{\mathrm{D}}{ }^{20} 1.5100\right)$ was synthesized and purified according to a previously developed technique. ${ }^{16} \mathrm{~N}$-vinylpyrrolidone (99\%, Sigma-Aldrich Co., St Louis, MO, USA) was used as a comonomer for the synthesis of new nontoxic copolymer. Poly (VT-co-VP) was synthesized by radical copolymerization of 1-vinyl-1,2,4-triazole and N-vinylpyrrolidone in dimethylformamide (DMF) under the action of $\alpha, \alpha^{\prime}$-azobisisobutyronitrile (AIBN) as an initiator $(1 \%$ from the monomer mass) at $60^{\circ} \mathrm{C}$ in an argon atmosphere (Figure 1). The synthesized hydrophilic poly (VT-co-VP) was used as the stabilizing agent for AgNPs. $\mathrm{AgNO}_{3}(99.9 \%$, Sigma-Aldrich) was used as a precursor for the formation of AgNPs. $\mathrm{NaBH}_{4}$ (Sigma-Aldrich) was used as the reducing agent. DMF, acetone, ethanol, and AIBN were distilled and purified according to the known procedures.

\section{Synthesis of poly (VT-co-VP)}

1-Vinyl-1,2,4-triazole(6.14 mL, 20.0 mmol), N-vinylpyrrolidone (3.20 mL, $20.0 \mathrm{mmol}), \mathrm{DMF}(1 \mathrm{~mL})$, and initiator AIBN $\left(2.0 \times 10^{-2} \mathrm{~mol} / \mathrm{L}\right)$ were placed in a tube. Then, the mixture was stirred and kept in a thermostat at $60^{\circ} \mathrm{C}$ for 1.5 hours until the formation of a transparent block (polymerization completion). The reaction mixture was dissolved in DMF, precipitated into acetone, and dried in vacuo over $\mathrm{P}_{2} \mathrm{O}_{5}$ at $50^{\circ} \mathrm{C}$ to a constant weight. The product was dissolved in water, purified by dialysis for 48 hours through a cellophane membrane with a pore size of $5 \mathrm{kDa}$ (Cellu Sep H1, MFPI, Seguin, USA), and freezedried to give the copolymer as a white powder. The product yield was higher than $84 \%$ and the polymer was soluble in water and dipolar organic solvents (DMF and DMSO). The molecular weight of the synthesized copolymer was $43 \mathrm{kDa}$. Further, the obtained copolymer was used for the subsequent synthesis of the metal polymer nanocomposite.

\section{Synthesis of AgNPs nanocomposites}

To a solution of poly (VT-co-VP) $(4.11 \mathrm{~g}, 39.6 \mathrm{mmol})$ in water $(40 \mathrm{~mL})$, an aqueous solution $(10 \mathrm{~mL})$ containing $\mathrm{AgNO}_{3}(0.34 \mathrm{~g}, 2.0 \mathrm{mmol})$ was added. The mixture was stirred intensively for 60 minutes at room temperature. Aqueous solution ( $5 \mathrm{~mL}$ ) containing $\mathrm{NaBH}_{4}(0.12 \mathrm{~g}, 3.0 \mathrm{mmol})$ was then added dropwise over 5 minutes, and the mixture 
was stirred further for 4 hours at room temperature. The resulting solution was precipitated into ethanol. The product was then filtered from ethanol, purified by dialysis against water through a cellophane membrane ( $5 \mathrm{kDa}$ cut-off), and freeze-dried to give nanocomposites as a dark-brown powder. The product yield was $75 \%-85 \%$ and the nanocomposite was soluble in water and dipolar organic solvents (DMF and DMSO). The silver content in nanocomposite was $9.0 \%$. The silver content can be easily controlled by varying the initial molar ratio of poly (VT-co-VP) and $\mathrm{AgNO}_{3}$. When the molar ratios of copolymer to silver salts were 15:1 and $10: 1$, the silver content was $12.3 \%$ and $17.7 \%$, respectively. This increase in the silver content led first to partial and then to a complete loss of solubility in all solvents.

\section{Characterization}

Fourier transform infrared (FTIR) spectra were recorded on a Vertex 70 spectrometer with $\mathrm{KBr}$ pellets (Bruker Corporation, Billerica, MA, USA). Ultraviolet-visible spectra were run on a Lambda 35 instrument (PerkinElmer Inc., Waltham, MA, USA). The silver content in the nanocomposites was determined using the atomic absorption analysis method (AAnalyst 200 instrument, PerkinElmer Inc.). Microphotographs were obtained using a transmission electron microscope (Leo 906E, Carl Zeiss AG, Oberkochen, Germany). X-ray diffraction patterns were obtained on a powder diffractometer (D8 Advance, Bruker Corporation). Thermogravimetric analysis and differential scanning calorimetry were performed using an STA 449 Jupiter derivatograph (Netzsch, Selb, Germany) in an atmosphere of air at a heating rate of $10^{\circ} \mathrm{C}$ per min from $25^{\circ} \mathrm{C}$ to $700^{\circ} \mathrm{C}$; the weight of the samples was $5 \mathrm{mg}$. The sedimentation stability of the sols was evaluated visually and by ultraviolet-visible spectra. Gel permeation chromatography (GPC) measurements were carried out using a PL-GPC 220 fitted with a differential refractive index detector (Agilent High Temperature Chromatograph). Dissolution of samples was performed at $80^{\circ} \mathrm{C}$ for 12 hours using a system for sample preparation Varian PL-SP 260VS. DMFA solution was used as an eluent with a flow rate of $1 \mathrm{~mL} /$ minute. Calibration was carried out using a series of polystyrene standards.

\section{Toxicity evaluation of poly (VT-co-VP)}

The studies were performed on 48 outbred white mice (24 males and 24 females) with a weight of 180-200 g. The animals were divided into three groups containing 16 animals each (eight males and eight females). The animals of the first group were administered with poly (VT-co-VP), and the animals of the second group received the silver containing nanocomposite in a preparative dose of $5,000 \mathrm{mg} / \mathrm{kg}$ in $0.5 \mathrm{~mL}$ of a saline solution. The animals of the third (control) group were administered with a saline solution in equivalent doses. The administration of the nanocomposite containing AgNPs to white mice before feeding was single and intragastric (with a stomach pump). The duration of observations of experimental animals after administration was 14 days.

All animals were divided into groups by the randomization method. The absence of external indications of diseases and homogeneity over the body weight were considered as criteria of appropriate randomization. The operation with laboratory animals was performed according to the Guiding Principles for Biomedical Research Involving Animals (Geneva, 1985), the Helsinki Declaration on the Human Attitude to Animals (Helsinki, 2000), the European Convention for the Protection of Vertebrate Animals Used for Experimental and Other Scientific Purposes (Strasbourg, 1986), and the Rules for Laboratory Practice (order no 708 n of the Ministry of Health Protection and Social Development of the Russian Federation of August 23, 2010).

\section{Antimicrobial activity}

The minimum inhibitory concentration and minimum bactericidal concentration (MIC and MBC) of the synthesized nanocomposite containing 9.0\% AgNPs was studied against Escherichia coli (ATCC25922), Pseudomonas aeruginosa (ATCC27853), Klebsiella pneumoniae (ATCC700603), Staphylococcus aureus (ATCC25923), and Enterococcus faecalis (ATCC29212) using a serial dissolution method. ${ }^{17}$ The main solutions of the nanocomposites contained $1,000 \mu \mathrm{g}$ in $1 \mathrm{~mL}$. Under aseptic conditions, these solutions were used to obtain two-fold concentrations of preparations in liquid feeding medium in a series of eight to eleven tubes $(1 \mathrm{~mL}$ volume) with a final concentration of the microorganism of $5 \times 10^{5} \mathrm{CFU} / \mathrm{mL}$. Antimicrobial activity was studied upon dissolution of samples in a range of $0.125-500 \mu \mathrm{g} / \mathrm{mL}$.

Suspensions of test organisms in a concentration of 0.5 McFarland standard $\left(1.5 \times 10^{8} \mathrm{CFU} / \mathrm{mL}\right)$ prepared from an agar culture in a sterile isotonic sodium chloride solution with a densitometer Densimat (Bio Merieux, Marcy-l'Étoile, France). From the microbial suspension, a series of consequent cultures 1:10 were prepared in a concentration of $10^{6} \mathrm{CFU} / \mathrm{mL}$. To obtain the necessary inoculum $\left(5 \times 10^{5} \mathrm{CFU} / \mathrm{mL}\right)$, in every tube $50 \mu \mathrm{L}$ of bacterial suspension containing $10^{6} \mathrm{CFU} / \mathrm{mL}$ was added. The control tube contained $1 \mathrm{~mL}$ of broth without a preparation and $50 \mu \mathrm{L}$ of the culture for each tested strain. The experiments were conducted twice for each culture (Table 1). 
Table I Antimicrobial activity of nanocomposite

\begin{tabular}{|c|c|c|c|c|c|c|c|c|c|}
\hline \multirow[t]{3}{*}{ Microorganisms } & \multicolumn{9}{|c|}{$\mathrm{MIC} / \mathrm{MBC}, \mu \mathrm{g} / \mathrm{mL}$} \\
\hline & $500-32$ & 16 & 8 & 4 & 2 & I & 0.5 & 0.25 & 0.12 \\
\hline & MIC/MBC & MIC/MBC & MIC/MBC & MIC/MBC & $M I C / M B C$ & MIC/MBC & MIC/MBC & MIC/MBC & MIC/MBC \\
\hline $\begin{array}{l}\text { Escherichia coli } \\
\text { ATCC } 25922\end{array}$ & $--I--$ & $--I--$ & $--I--$ & $--I--$ & $--I--$ & $--I--$ & $--I--$ & $++/++$ & $++/++$ \\
\hline $\begin{array}{l}\text { Pseudomonas aeruginosa } \\
\text { ATCC } 27853\end{array}$ & $--I--$ & $--I--$ & $--I--$ & $--I--$ & $--l++$ & $--1++$ & $++/++$ & $++/++$ & $++/++$ \\
\hline $\begin{array}{l}\text { Klebsiella pneumoniae } \\
\text { ATCC } 700603 \text { (EBSL) }\end{array}$ & $--I--$ & $--I--$ & $--1--$ & $--1--$ & $--1++$ & $++/++$ & $++/++$ & $++/++$ & $++/++$ \\
\hline $\begin{array}{l}\text { Staphylococcus aureus } \\
\text { ATCC } 25923\end{array}$ & $--1--$ & $--1--$ & $--I--$ & $--I--$ & $--l++$ & $++/++$ & $++/++$ & $++/++$ & $++/++$ \\
\hline $\begin{array}{l}\text { Enterococcus faecalis } \\
\text { ATCC } 29212\end{array}$ & $--I--$ & $--I--$ & $--l++$ & $++/++$ & $++/++$ & $++/++$ & $++/++$ & $++/++$ & $++/++$ \\
\hline
\end{tabular}

Notes: (-) Absence of test-strain growth; (+) presence of growth of test strains.

Abbreviations: MBC, minimum bactericidal concentration; MIC, minimum inhibitory concentration; ATCC, American Type Culture Collection.

The inocula were incubated under common atmosphere at $35^{\circ} \mathrm{C}$ for $18-24$ hours. The results were evaluated visually determining presence or absence of growth in the medium containing the studied compound in different concentrations. The last tube with growth retardation (transparent broth) corresponded to the minimum inhibiting concentration of the preparations with respect to a strain.

To determine cell viability, the cultures from all transparent tubes were inoculated on a solid medium (Mueller-Hinton agar). After incubation of inocula in thermostat, the lowest concentration of the preparation in the tube was determined. This concentration was referred to as MBC.

\section{Results and discussion}

\section{Copolymerization of VT with VP}

Radical copolymerization of 1-vinyl-1,2,4-triazole with $\mathrm{N}$-vinylpyrrolidone was carried out in DMF solution in the presence of an initiator (AIBN) at $60^{\circ} \mathrm{C}$ for 1.5 hours, molar ratio of the starting monomers being 70:30 (Figure 1).

The copolymer obtained (white powder) is well soluble in water and dipolar organic solvents. Composition and structure of the copolymer were established by Fourier Transform Infrared spectroscopy (FTIR), ${ }^{1} \mathrm{H}$, ${ }^{13} \mathrm{C}$ Nuclear Magnetic Resonances spectroscopy, and elemental analysis data. The content of the triazole and pyrrolidone fragments in the copolymer was 49 and 51 mol\%, respectively.

The GPC traces for poly (VT-co-VP) were obtained using polystyrene standards. The number average (Mn) and the weight average $(\mathrm{Mw})$ molecular weights of the polymer were measured to be 29,541 and 57,119 , respectively. The polydispersity index $(\mathrm{Mw} / \mathrm{Mn})$ of the copolymer was 2.1 .

\section{Nanocomposite}

Mixing of poly (VT-co-VP) and $\mathrm{AgNO}_{3}$ aqueous solutions during the first stage in synthesis of nanocomposite led to an increase in viscosity of the solution, and viscous cream-like gels were formed. On vigorous stirring and stepwise addition of $\mathrm{NaBH}_{4}$, the cream-like gels were transformed into darkcolored sols, from which the hydrophilic nanocomposite was isolated. Analysis of the elemental data and atomic absorption spectroscopy showed that the silver content in nanocomposite was $9.0 \%$. The isolated and purified polymer nanocomposites are readily soluble in water and dipolar organic solvents (DMF and DMSO).

The solubility of the nanocomposites is mainly due to the intramolecular interaction of copolymer macromolecules with AgNPs, which form relatively loose polymer coils unfolded somewhat in an aqueous solution because there are not many nanoparticles involved in the coordination interactions

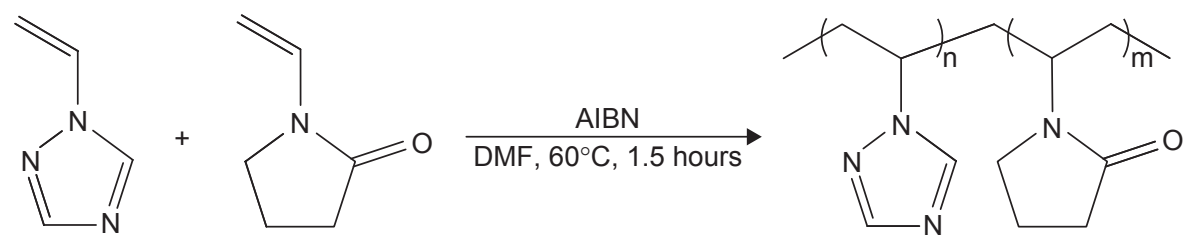

Figure I Synthesis of poly (VT-co-VP).

Abbreviations: AIBN, $\alpha, \alpha^{\prime}$-azobisisobutyronitrile; DMF, dimethylformamide; poly (VT-co-VP), copolymer of I-vinyl-I,2,4-triazole and $\mathrm{N}$-vinylpyrrolidone. 


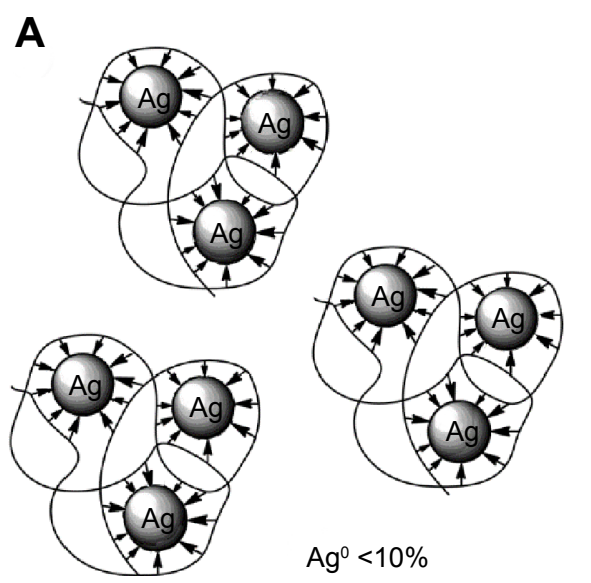

B

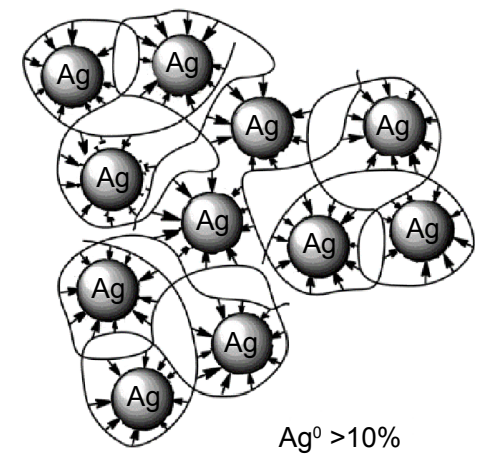

Figure 2 Intramolecular (A) and intermolecular (B) interactions of silver nanoparticles with poly (VT-co-VP) macromolecules. Abbreviation: Poly (VT-co-VP), copolymer of I-vinyl-I,2,4-triazole and N-vinylpyrrolidone.

(Figure 2A). A polymer coil is a more compact conformational state of a polymer nanocomposite macromolecule than that of the initial copolymer, whose state results from partial crosslinking by the nanosized particles. An increase in the silver content above $10 \%$ (at copolymer-silver salt molar ratios of 15:1 and 10:1) leads, first, to partial and then to complete loss of solubility. This loss is owing to enhancement of the intermolecular interaction due to cross-linking of polymer macromolecules with metal nanoparticles via the action of multiple cooperative forces (Figure 2B). Intermolecular binding of macromolecules by surface atoms of AgNPs significantly competes with the copolymer hydration process because the solubility of the copolymers is due to the hydrogen bonds of the triazole and lactam rings. Thus, with an increase in the concentration of AgNPs, the hydration of the polymer matrix nanocomposite will progressively become more difficult until its complete loss (most of the triazole and lactam rings are involved in the coordination interaction with the AgNPs and do not participate in hydrogen bonding with water molecules).

The optical absorption spectrum of nanocomposite in aqueous solution, unlike the initial poly (VT-co-VP) and $\mathrm{AgNO}_{3}$ aqueous solutions, shows a characteristic plasmon resonance absorption band of conductivity electrons from the metallic AgNPs. The time evolution of the ultraviolet-visible absorption spectra is shown in Figure 3. After 5 minutes of the addition of the reducing agent at vigorous stirring, a brown colloidal solution is formed, with an absorption peak at $398 \mathrm{~nm}$ (Figure 3), indicating the initial stage of generation of small metallic AgNPs. Increasing the reaction time leads to formation of a homogeneous dark-brown sol. Reduction of the silver ions is accompanied by diminution of the intensity of the absorption band due to decreased content and a red-shift of the peak position from 398 to
$410 \mathrm{~nm}$ owing to increase in size of AgNPs. Recovery and growth of the AgNPs are completed within 4 hours, with the ultraviolet-visible absorption curves recorded after 4 and 6 hours being almost superimposable. The aqueous poly (VTco-VP)-stabilized AgNPs dispersion is centrifuged at 10,000× $g$ for 15 minutes. A small amount of the precipitate is obtained from the bottom of the centrifuge tube and is found to be completely soluble in aqueous solution again after shaking of the centrifuge tube. Absorption bands of AgNPs taken before and after centrifugation are identical, which indicates a high stabilizing capability of the polymer matrix.

The scanning electron microscopy (SEM) images of poly (VT-co-VP)copolymer and nanocomposite with AgNPs support their different surface morphology. It has been shown that the initial copolymer has highly porous spongelike surface with numerous channels $2-6 \mu \mathrm{m}$ in diameter (Figure 4A). In the case of AgNPs nanocomposites, the surface morphology becomes denser and porous structure is still observed on an SEM image (Figure 4B).

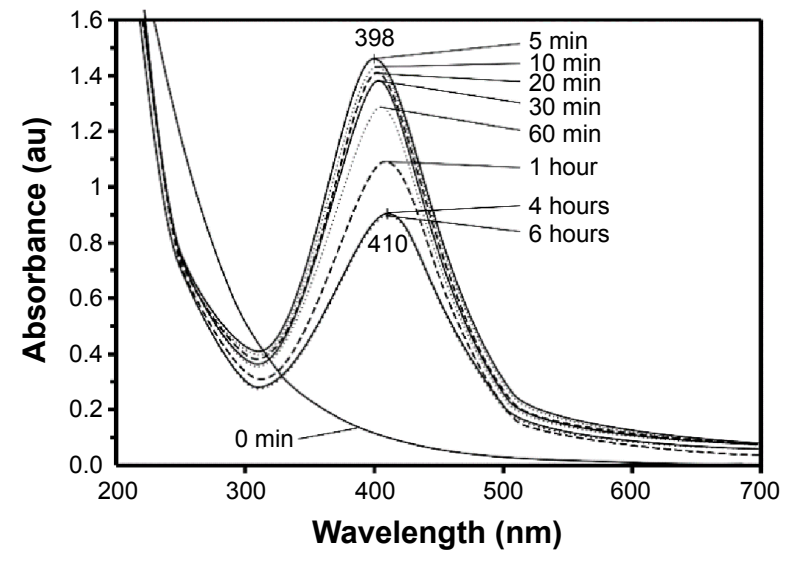

Figure 3 Time evolution of the ultraviolet-visible absorption spectra for nanocomposite. 

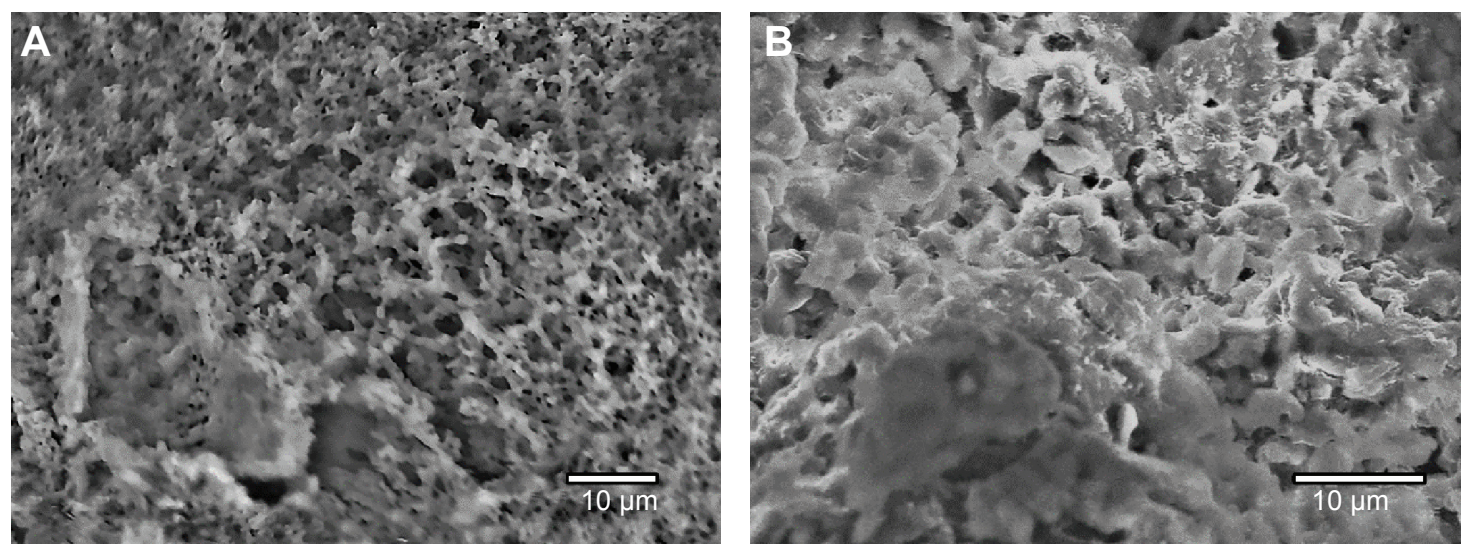

Figure 4 SEM microphotographs of poly (VT-co-VP) copolymer (A) and silver nanoparticles nanocomposite (B).

Notes: Magnification (A) 2000x; magnification (B) 3000x.

Abbreviation: SEM, scanning electron microscopy; poly (VT-co-VP), copolymer of I-vinyl-I,2,4-triazole and N-vinylpyrrolidone.

The morphology and size distribution of the AgNPs obtained have been studied by transmission electron microscopy. The transmission electron micrographs and histograms for the synthesized AgNPs are shown in Figure 5. As can be seen, spherical AgNPs of 2-6 nm in size are formed and stabilized in the poly (VT-co-VP) matrix. The distribution of nanoparticles in the polymer matrix is homogeneous. Most (94\%) of the AgNPs have a diameter of 2-5 nm (Figure 5). Such narrow distribution of the nanoparticles evidences efficient stabilization of AgNPs at the first stages of their formation due to the high coordination ability of the triazole and lactam rings.

\section{X-ray diffraction and FTIR characterization}

A typical X-ray diffractogram for the AgNPs nanocomposites stabilized by poly (VT-co-VP) is shown in Figure 6A, where the amorphous halo of the polymer matrix and reflexes of the zerovalent metallic silver are clearly differentiated. Three main characteristic diffraction peaks for silver are observed at $2 \theta 38.2,44.3$, and 64.5 , corresponding to the (111), (200), and (220) crystalline planes of the face-centered cubic crystalline structure of metallic silver. The latter is identified by comparison of the values for interplanar spacing and relative intensities with the standard values for metal silver. X-ray diffraction spectrum of AgNPs is matched with JCPDS card No 04-0783. An X-ray diffraction study demonstrates that the average size of the AgNPs (average area of coherent scattering), determined by the Debye-Scherrer method, is 46-48 $\AA$. The amorphous phase of the polymer component of the nanocomposites is determined by comparison with the diffractogram of poly (VT-co-VP) (Figure 6B).

FTIR spectroscopy has been used to study the interactions between the polymeric matrix and the AgNPs. Figure 7 depicts the FTIR spectra of the starting poly (VT-co-VP) copolymer and nanocomposite. The stretching and deformation vibrations of the initial polymer rings are observed for the triazole ring at 3,111 $(\mathrm{C}-\mathrm{H}), 1,502(\mathrm{C}=\mathrm{N}), 1,425$
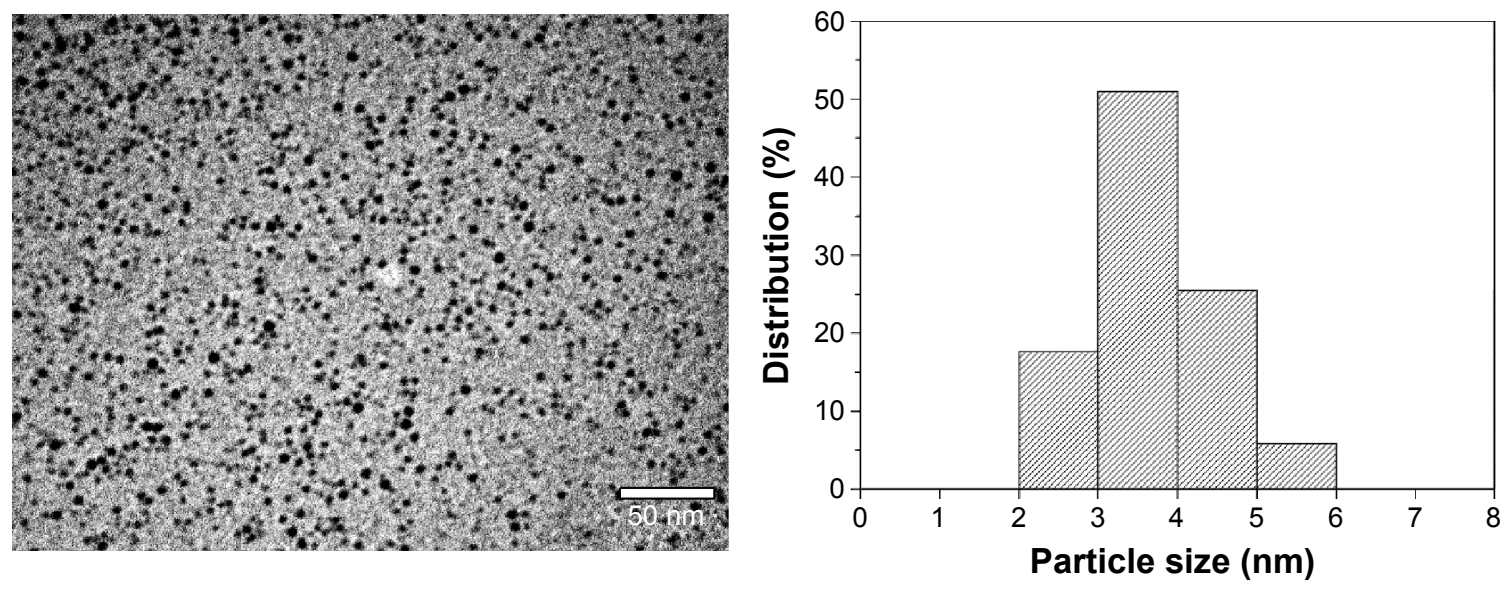

Figure 5 Transmission electron micrograph and respective histogram of silver nanoparticle nanocomposite. 


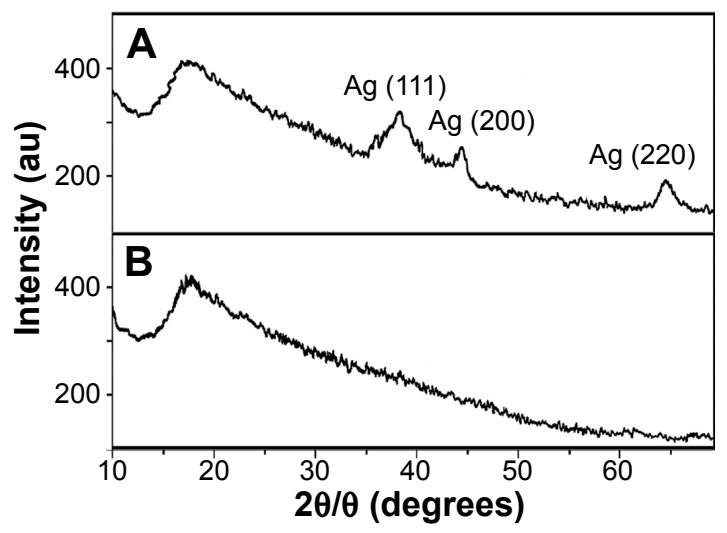

Figure 6 X-ray diffraction pattern for nanocomposite with silver nanoparticles (A) and the starting poly (VT-co-VP) (B).

Abbreviation: poly (VT-co-VP), copolymer of I-vinyl-1,2,4-triazole and $\mathrm{N}$-vinylpyrrolidone.
$(\mathrm{C}-\mathrm{N}), 1,271(\mathrm{~N}-\mathrm{N}), 1,140(\mathrm{C}-\mathrm{H}), 1,005(\mathrm{C}-\mathrm{H})$, and 667 $(\mathrm{C}-\mathrm{N}) \mathrm{cm}^{-1}$, and for the lactam ring at 1,678 $(\mathrm{C}=\mathrm{O})$ and $1,290(\mathrm{C}-\mathrm{N}) \mathrm{cm}^{-1}$. The FTIR spectrum of the nanocomposite is similar to that of the initial copolymer. This testifies to the absence of structural transformations in poly (VT-co-VP) during synthesis of the nanocomposites and indicates that the original properties of the polymer matrix are retained. Also, the bands corresponding to vibrations of the triazole and lactam rings are shifted from 1,678, 1,502, 1,425, 1,290, and $1,271 \mathrm{~cm}^{-1}$ to $1,664,1,508,1,435$, and $1,278 \mathrm{~cm}^{-1}$ (Figure 7). This evidences the coordination interactions of $\mathrm{N}$ and $\mathrm{O}$ atoms of the triazole and lactam rings with AgNPs.

The presence of poly (VT-co-VP) in the reaction mixture promotes coordinated interaction (at the reduction

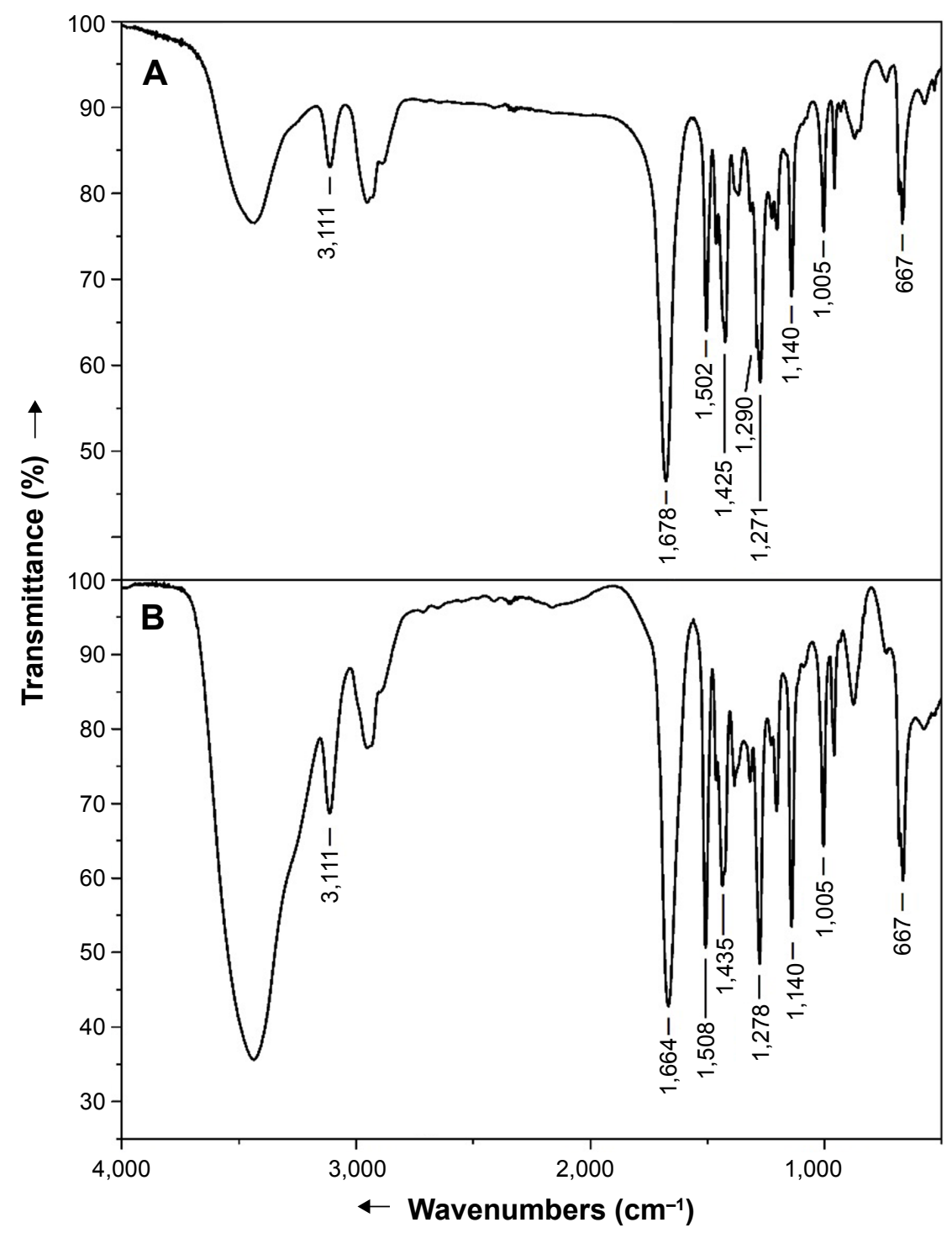

Figure 7 Fourier transform infrared spectra for the starting poly (VT-co-VP) (A) and nanocomposite (B). Abbreviation: poly (VT-co-VP), copolymer of I-vinyl-1,2,4-triazole and $\mathrm{N}$-vinylpyrrolidone. 
stage) between AgNPs and the coordination rings (triazole and lactam) in the copolymer. It prevents their further agglomeration and ensures their homogeneous distribution throughout the copolymer matrix. The aqueous solutions containing AgNPs nanocomposites were allowed to stand for an extended time in air at room temperature, and no signs of sedimentation were observed even after 4 months of storage. This indicates that the AgNPs synthesized in this polymer matrix are stable and that the hydrophilic poly (VT-co-VP) has a high stabilizing ability.

\section{Thermogravimetric analysis}

The results of thermogravimetric analysis and differential scanning calorimetry show that the initial poly (VT-co-VP) has thermal stability up to $320^{\circ} \mathrm{C}$ (Figure 8). The thermal stability of nanocomposite decreases by $50^{\circ} \mathrm{C}$ compared with that of the initial polymer, and thermal destruction of the nanocomposites starts at $270^{\circ} \mathrm{C}$. In the paper, ${ }^{18}$ the authors also observed decrease in thermal stability of the polymer due to the presence of silver particles in nanocomposites.

For nanocomposite, it has been found that the adsorbed water is released at $60^{\circ} \mathrm{C}-220^{\circ} \mathrm{C}$ (weight loss from the sample is $8.1 \%$ ), and this is accompanied by an endothermic effect in the differential scanning calorimetry curve. This is supported by the finding of a signal in the mass spectrum at a mass number of 18 . The second and third stages of thermal destruction of the polymeric component are observed at $270^{\circ} \mathrm{C}-410^{\circ} \mathrm{C}$ as evidenced from the presence of two exothermic effects (weight loss from the sample is $45.7 \%$ ). This is accompanied by abstraction of the triazole and lactam fragment from the macromolecular chain with subsequent oxidation to $\mathrm{H}_{2} \mathrm{O}$, $\mathrm{CO}_{2}$, and $\mathrm{NO}_{2}$ that is confirmed by appearance of signals in the mass spectrum at the corresponding mass numbers $(18,44$, and 46$)$. At $480^{\circ} \mathrm{C}-570^{\circ} \mathrm{C}$, weight loss from the sample reaches $41.9 \%$ and the intensive exothermic effect at a maximum at $546.7^{\circ} \mathrm{C}$ takes place, corresponding to burning of the carbon skeleton, to yield mainly $\mathrm{CO}_{2}$ (mass number 44). In the differential scanning calorimetry curve, the endothermic effect responsible for melting of metallic silver is detected at $961.7^{\circ} \mathrm{C}$.

The decreased thermal stability of the nanocomposites is caused by the catalytic peculiarities of AgNPs, which decrease the activation energy of thermal destruction and oxidation of the polymer matrix. The weight loss or difference in decomposition between the copolymer and silver nanocomposite is found to be $6.8 \%$ thus indicating the presence of AgNPs (weight loss) in the poly (VT-co-VP).

\section{Toxicity evaluation of poly (VT-co-VP)}

Good endurance of these drugs by animals (in the absence of intoxication and death symptoms) was established when determining the toxicity of the initial poly (VT-co-VP) and nanocomposite with AgNPs. No differences in weight, sensitivity to the studied drugs by sex, and deviations in feed and drink consumption between tested and control animals were observed. The macroscopic postmortem examination of animals of the first (poly (VT-co-VP) administered), second (the silver-containing nanocomposite administered), and third (control) groups sacrificed at the end of the experiment (in 14 days) revealed no differences. The internal organs were without changes, and no extravasations were observed. The analysis of the obtained data suggested that the lethal dose $\mathrm{LD}_{50}$ for poly (VT-co-VP) and related silver-containing nanocomposite is higher than $5,000 \mathrm{mg} / \mathrm{kg}$.

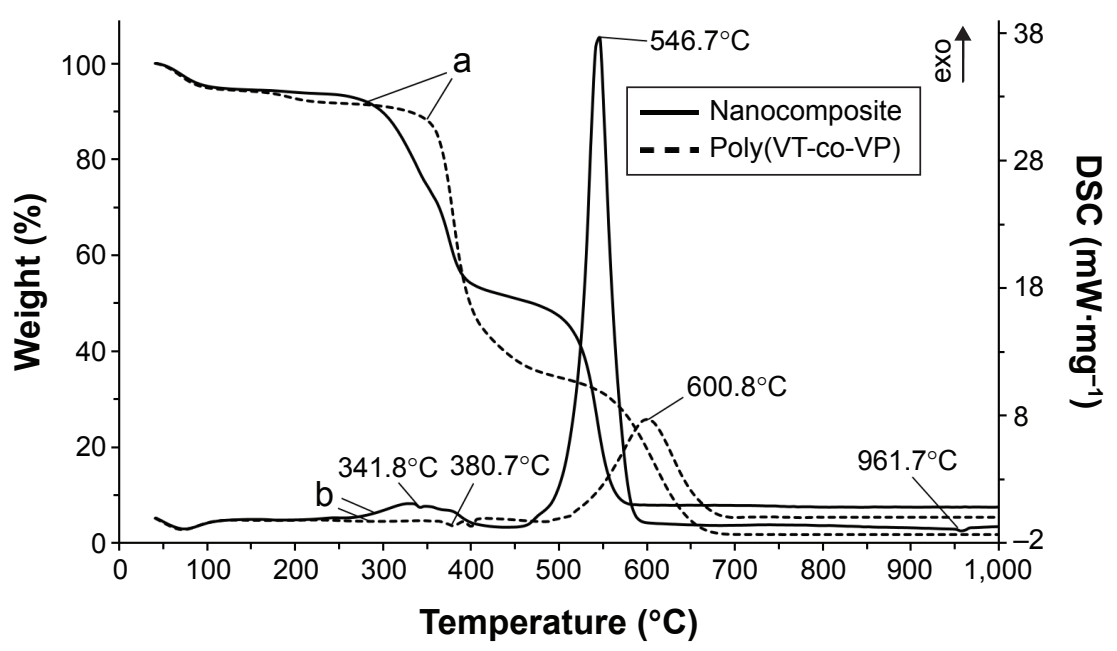

Figure 8 Thermogravimetric (a) and differential scanning calorimetry (b) curves for the starting poly (VT-co-VP) and nanocomposite. Abbreviations: DSC, differential scanning calorimetry; exo, exothermic effects; poly (VT-co-VP), copolymer of I-vinyl-I,2,4-triazole and N-vinylpyrrolidone. 


\section{Antimicrobial activity}

Microbiological examination has shown that the nanocomposite has a pronounced antimicrobial effect toward different strains of Gram-negative and -positive bacteria and Gramnegative organisms are more sensitive than Gram-positive (Table 1). It has been reported ${ }^{19}$ that Gram-negative bacteria are more sensitive to the action of silver than Gram-positive ones. Our study also confirms the relationship between antimicrobial activity of the nanocomposite and cell walls of Gram-positive and -negative bacteria having a different structure. In Gram-negative bacteria, rigid layer with hydrophilic channels $(\sim 2-3 \mathrm{~nm})$ probably ensures the best linking with the silver nanocomposite than in the case of Gram-positive bacteria $(20-60 \mathrm{~nm})$.

The MICs suppressing the growth of the microorganisms range from 0.5 to $8 \mu \mathrm{g} / \mathrm{mL}$ and the MBCs from 0.5 to $16 \mu \mathrm{g} / \mathrm{mL}$. E. coli (ATCC25922) is the most sensitive microorganism among all investigated, for its $\mathrm{MIC}$ and $\mathrm{MBC}$ coincides $(0.5 \mu \mathrm{g} / \mathrm{mL})$. For other Gram-negative bacteria, $P$. aeruginosa (ATCC27853), MIC is $1 \mu \mathrm{g} / \mathrm{mL}$ and MBC is $4 \mu \mathrm{g} / \mathrm{mL}$. For microorganisms K. pneumoniae (ATCC700603) (extendedspectrum $\beta$-lactamase) and $S$. aureus (ATCC25923), the minimum antimicrobial concentration coincides MIC is $2 \mu \mathrm{g} / \mathrm{mL}$ and $\mathrm{MBC}$ is $4 \mu \mathrm{g} / \mathrm{mL}$. The best values of MIC and MBC ( 8 and $16 \mu \mathrm{g} / \mathrm{mL}$, respectively) the synthesized nanocomposite has shown against Gram-positive test microorganisms E. faecalis (ATCC29212). The data of the initial studies of antimicrobial activity of the starting poly (VT-co-VP) show no effect at the same concentrations.

The results obtained are indicative of higher antimicrobial activity of AgNPs nanocomposites in the matrix of the poly (VT-co-VP) as compared to silver-containing nanocomposites based on 1-vinyl-1,2,4-triazole homopolymer. ${ }^{8}$

\section{Conclusion}

In this study, we have reported a synthesis of new hydrophilic nontoxic nanocomposites containing AgNPs in a functional polymer matrix of poly (1-vinyl-1,2,4-triazole-co$\mathrm{N}$-vinylpyrrolidone). The reduction of silver ions in the presence of copolymer containing triazole and lactam groups, as the stabilizing matrix, leads to the formation of monodispersed AgNPs with a narrow size distribution (2-6 nm). The AgNP nanocomposites obtained are thermally stable up to $270^{\circ} \mathrm{C}$ and highly stable. Due to the effective stabilization by functional groups, AgNPs do not precipitate and or change in size even after storage in aqueous solutions for 4 months. The toxicity of the initial poly (VT-co-VP) copolymer and nanocomposite containing AgNPs has been determined on white mice, its value being more than $5,000 \mathrm{mg} / \mathrm{kg}$. The nanocomposite had pronounced antimicrobial activity toward different strains of Gram-negative and -positive bacteria. The MICs suppressing the growth of the microorganisms range from 0.5 to $8 \mu \mathrm{g} / \mathrm{mL}$ and the $\mathrm{MBC}$ range from 0.5 to $16 \mu \mathrm{g} / \mathrm{mL}$. Therefore, these new AgNPs nanocomposites in a poly (VT-co-VP) matrix are promising materials for the design of novel nontoxic hydrophilic antiseptics and antimicrobial components for medical purposes for the treatment of many infectious diseases, including surgical ones.

\section{Acknowledgment}

This study was supported by a grant from the Russian Foundation of Basic Research (15-03-08295).

\section{Disclosure}

The authors report no conflicts of interest in this work.

\section{References}

1. Luo ZT, Zheng KY, Xie JP. Engineering ultrasmall water-soluble gold and silver nanoclusters for biomedical applications. Chem Commun(Camb). 2014;50(40):5143-5155.

2. Yao J, Yang M, Duan YX. Chemistry, biology, and medicine of fluorescent nanomaterials and related systems: new insights into biosensing, bioimaging, genomics, diagnostics, and therapy. Chem Rev. 2014 114(12):6130-6178

3. Rai M, Yadav A, Gade A. Silver nanoparticles as a new generation of antimicrobials. Biotechnol Adv. 2009;27(1):76-83.

4. Prozorova GF, Pozdnyakov AS, Korzhova SA, et al. Toxicity evaluation of polyvinyltriazole and a related silver-containing nanocomposite. Russ Chem Bull. 2014;63(9):2126-2129.

5. Pozdnyakov AS, Emel'yanov AI, Ermakova TG, Prozorova GF. Functional polymer nanocomposites containing triazole and carboxyl groups. Polym Sci Ser B. 2014;56(2):238-246.

6. Khutsishvili SS, Vakul'skaya TI, Kuznetsova NP, Ermakova TG, Pozdnyakov AS, Prozorova GF. Formation of stable paramagnetic nanocomposites containing zero-valence silver and copper in a polymeric matrix. J Phys Chem C. 2014;118(33):19338-19344.

7. Pozdnyakov AS, Kuznetsova NP, Korzhova SA, et al. Antimicrobial activity of $\mathrm{Ag}(0)$-nanocomposite copolymer of 1-vinyl-1,2,4-triazole with acrylic acid sodium salt. Russ Chem Bull. 2015;64(6):1440-1444.

8. Prozorova GF, Pozdnyakov AS, Kuznetsova NP, et al. Green synthesis of water-soluble nontoxic polymeric nanocomposites containing silver nanoparticles. Int J Nanomed. 2014;9:1883-1889.

9. Kopeikin VV, Panarin EF. Water-soluble nanocomposites of zerovalent metallic silver with enhanced antimicrobial activity. Dokl Chem 2001;380(4-6):277-279.

10. Gorbunova M, Lemkina L. New biocide guanidine-containing nanocomposites. J Nanopart Res. 2014;16:2566.

11. Lu Z, Rong K, Li J, Yang H, Chen R. Size-dependent antibacterial activities of silver nanoparticles against oral anaerobic pathogenic bacteria. J Mater Sci Mater Med. 2013;24(6):1465-1471.

12. Chernousova S, Epple M. Silver as antibacterial agent: ion, nanoparticle, and metal. Angew Chem Int Ed Engl. 2013;52(6):1636-1653.

13. Wang LS, Wang CY, Yang CH, et al. Synthesis and anti-fungal effect of silver nanoparticles-chitosan composite particles. Int J Nanomedicine. 2015;10:2685-2696.

14. Korbekandi H, Iravani S. Silver nanoparticles. In: Hashim AA, editor The Delivery of Nanoparticles. Rijeka, Croatia: InTech; 2012:1-35. 
15. Mecha CA, Pillay VL. Development and evaluation of woven fabric microfiltration membranes impregnated with silver nanoparticles for potable water treatment. J Membrane Sci. 2014;458: 149-156.

16. Ermakova TG, Tatarova LA, Kuznetsova NP. Vinylation of 1,2,4triazole. Russ J Gen Chem. 1997;67(5):859-861.

17. Fisenko VP. Guidance on experimental (preclinical) study of novel pharmacological substances. Moscow: IIA Remedium; 2000.
18. Lee K, Rah S, Kim S-G. Formation of monodisperse silver nanoparticles in poly(vinylpyrrollidone) matrix using spray pyrolysis. $J$ Sol-Gel Sci Techn. 2008;45(2):187-193.

19. Gurunathan S, Han JW, Kwon DN, Kim JH. Enhanced antibacterial and anti-biofilm activities of silver nanoparticles against Gram-negative and Gram-positive bacteria. Nanoscale Res Lett. 2014;9:373.

\section{Publish your work in this journal}

The International Journal of Nanomedicine is an international, peerreviewed journal focusing on the application of nanotechnology in diagnostics, therapeutics, and drug delivery systems throughout the biomedical field. This journal is indexed on PubMed Central, MedLine, CAS, SciSearch $®$, Current Contents ${ }^{\circledR} /$ Clinical Medicine,
Journal Citation Reports/Science Edition, EMBase, Scopus and the Elsevier Bibliographic databases. The manuscript management system is completely online and includes a very quick and fair peer-review system, which is all easy to use. Visit http://www.dovepress.com/ testimonials.php to read real quotes from published authors. 